\title{
Magnification endoscopy with optical chromoendoscopy shows strong correlation with histologic inflammation in patients with inflammatory bowel disease $\square$
}

\section{다)(1) $\odot(9$}

\author{
Authors \\ Entcho Klenske ${ }^{1}$, Raja Atreya ${ }^{1}$, Arndt Hartmann ${ }^{2}$, Sarah Fischer ${ }^{1}$, Simon Hirschmann ${ }^{1}$, Sebastian Zundler ${ }^{1}$, Marietta \\ laccuci $^{3}$, Markus F. Neurath ${ }^{1}$, Timo Rath ${ }^{1}$
}

Institutions

1 Department of Medicine I, Division of Gastroenterology, Ludwig Demling Endoscopy Center of Excellence, University Hospital of Erlangen, University ErlangenNürnberg, Germany

2 Institute of Pathology, University Hospital of Erlangen, University Erlangen-Nürnberg, Germany

3 Institute of Translational Medicine, University of Birmingham, United Kingdom

submitted 27.7.2018

accepted after revision 24.4.2019

\author{
Bibliography \\ DOI https://doi.org/10.1055/a-0953-1334 | \\ Endoscopy International Open 2019; 07: E1018-E1026 \\ (c) Georg Thieme Verlag KG Stuttgart · New York \\ eISSN 2196-9736
}

\section{Corresponding author}

Timo Rath, MD, Professor of Endoscopy and Molecular Imaging, Division of Gastroenterology, Ludwig Demling Endoscopy Center of Excellence, University Hospital of Erlangen, Ulmenweg 18, 91054 Erlangen, Germany Fax: +49 91318535272

Timo.Rath@uk-erlangen.de

\section{ABSTRACT}

Background and study aims Apart from mucosal healing as an established treatment goal in inflammatory bowel diseases (IBD), recent evidence suggests that histologic healing may become another key prognostic parameter in
IBD patients. We aimed to evaluate whether magnification endoscopy with optical chromoendoscopy can accurately assess histologic inflammation in IBD patients.

Patients and methods In this prospective study, 82 patients with IBD ( 30 UC, 52 CD) were included. In all patients, magnification endoscopy in conjunction with optical chromoendoscopy was performed and rated on a novel magnification endoscopy score by three independent endoscopists. Targeted biopsies of the imaged areas were obtained and results were compared against two histological scores in UC (Robarts Histopathology Index, RHI; Nancy Histology Index, NHI) and one score in CD (modified Riley index, mRI). Moreover, interobserver agreement was calculated.

Results Magnification endoscopy showed strong correlation with histopathologic scoring in both $U C(R H I: r=0.83$, NHI: $r=0.78, P<0.05)$ and $C D(m R I: r=0.74, P<0.05)$ with high accuracy, sensitivity, and specificity. Further, $25 \%$ of patients with mucosal healing on standard endoscopy showed signs of microinflammation on magnification endoscopy with optical chromoendoscopy, while none of the patients with mucosal and vascular healing under magnification endoscopy with optical chromoendoscopy exhibited microscopic inflammation. Interobserver agreement for grading intestinal inflammation by magnification endoscopy with optical chromoendoscopy was substantial $(\mathrm{K}>0.7)$.

Conclusion Magnification endoscopy in combination with optical chromoendoscopy shows strong correlation with histologic inflammation in patients with IBD. This approach has potential to reduce physical biopsies for monitoring of inflammatory activity in patients with IBD during colonoscopy.

\section{Introduction}

Mucosal healing has been established as a key therapeutic goal in management of patients with inflammatory bowel disease (IBD) that is associated with improved long-term clinical outcomes, i.e. decreased hospitalization rate and need for corti- costeroid therapy [1-6]. Therefore, consensus guidelines for clinical practice and trial endpoints recommend achieving endoscopic mucosal healing in addition to clinical remission as a central treatment target $[7,8]$. However, substantial disparity between endoscopic and histological disease activity exists with several reports showing that white light endoscopy (WLE) 
cannot accurately define and detect persistent histologic inflammation [9-12]. At the same time, first evidence suggests that histological remission even better predicts need for corticosteroid use and hospitalization than mucosal healing on endoscopy $[13,14]$ and based on this evidence, histological healing has been proposed as another key prognostic parameter in IBD patients [15]. However, histological scoring currently is not standardized, 26 different histopathological scores exist for inflammatory assessment of UC of which only two are validated [16], and no score is completely representative of patients with CD [17], resulting in limitations on their direct clinical application.

Further, the process of routinely sending specimens for microscopic grading of inflammatory activity in clinical practice is cost- and time-intensive. Recently, initial studies have shown that magnification endoscopy in combination with optical chromoendoscopy such as NBI (Olympus, Tokyo, Japan) and iscan optical enhancement (i-scan OE, Pentax, Tokyo, Japan) can accurately identify microscopic inflammation in UC and show a good correlation with histopathological scoring in this disease $[18,19]$.

In this study we aimed to not only verify these results in an independent cohort but also to extend the finding for the first to time Crohn's disease. For this purpose, we assessed whether magnification endoscopy in combination with i-scan OE can detect histologic inflammation in a large cohort of patients with IBD with CD and UC using a newly developed magnification endoscopy score.

\section{Patients and methods}

\section{Patients}

This was a prospective observational study conducted from March 2017 to March 2018 at the Ludwig Demling Endoscopy Center of Excellence at the University Hospital of Erlangen. The study was approved by the local ethics committee as well as the Institutional Review Board of the Medical Faculty of the Friedrich-Alexander University Erlangen-Nuremberg. No minors were included and written informed consent was obtained from all patients prior to the procedure. Patients with poor bowel preparation and/or total colectomy were excluded. All colonoscopies were performed using a high-definition colonoscope with optical magnification (MagniView, Pentax, Tokyo, Japan) equipped with the Pentax Medical OPTIVISTA EPK-i7010 video processor (Pentax, Tokyo, Japan).

Extent of inflammation was described according to the Montreal classification [20]. Clinical disease activity for patients with UC and CD was determined using the Mayo clinical disease activity score and the Crohn's disease activity index (CDAI) [21], respectively. In addition, sociodemographic factors, current and past medication along with routine laboratory parameters were assessed at the time of colonoscopy.

\section{Endoscopic assessment}

Colonoscopies were performed by one colonoscopist experienced in EC and optical diagnosis in IBD (T.R.). Upon WLE examination, disease activity was scored using the Mayo endoscopic subscore (Mayo ES, range 0 - 3) [22] in patients with UC and the Simple Endoscopic Score for Crohn's Disease (SES-CD, range $0-60)$ in patients with CD [23]. Imaging was then switched to i-scan imaging and optical magnification was used to inspect the mucosa with 136-fold optical zoom. Evaluation of disease activity according to the introduced magnification score was done endoscopically in the segment of the colon with the most severe disease, from which biopsies were taken for direct comparison with histopathology. Images and videos under magnification endoscopy with optical chromoendoscopy from each patient were digitally stored used for subsequent analyses.

A novel magnification i-scan OE score for grading inflammatory in patients with UC and CD was developed on the basis of a previous report on magnification endoscopy in UC [19]. Briefly, in UC, colonic or rectal inflammation were assessed using a mucosal pattern (MP) and vascular pattern (MP) score, which were each graded on a scale of 1 to 4 , and the overall score was calculated as the sum of the mucosal and vascular scores as previously described [19], therewith ranging from 2 to 8 . In $C D$, apart from using the same mucosal and vascular scores to assess colonic or rectal inflammation, a separate score was developed to assess mucosal and vascular changes within the terminal ileum under magnification endoscopy in conjunction with optical chromoendoscopy. Development of the novel magnification score for the terminal ileum was based on the consensus of four IBD-experienced endoscopists (T.R., E.K., R.A. and M.F.N.), each of whom perform $>250$ IBD colonoscopies per year.

From the stored, anonymized magnification images and video sequences, post hoc assessment of endoscopic inflammation and grading was performed according to the newly developed scores as outlined above. This post-hoc assessment and grading of inflammatory activity was done independently by a total of three endoscopists blinded to patient characteristics and results of histopathological scoring. Finally, interobserver agreement was calculated using Kappa agreement statistics.

\section{Histopathological assessment}

All biopsies were taken from the same regions of interest (ROI) primarily visualized with i-scan OE. At least two biopsies sample were taken from each segment according to the standard colonoscopic protocol at our department. Histopathological evaluation was done by one experienced gastroinestinal pathologist (A.H.) blinded to results of endoscopic scoring. For histopathological scoring, UC Robarts Histopathology Index (RHI) [24] as well as Nancy histological index (NHI) [25] were used as validated histology scores. Because there is no validated score for grading for histological inflammation in patients with $C D$, we used a modification of the Riley score [26] as previously described by our group in CD [27]. This score represents a modified version of the common Riley score and apart from including the six histological features for ulcerative colitis (acute inflammatory cell infiltrate, crypt abscesses, mucin depletion, surface epithelial integrity, chronic inflammatory cell infiltrate, crypt architectural irregularities), this score integrates typical histologic features observed in CD, namely lymphocyte aggre- 
- Table1 Demographic and clinical patient characteristics.

\begin{tabular}{|c|c|c|}
\hline & $\begin{array}{l}\text { Ulcerative } \\
\text { colitis }(n=30)\end{array}$ & $\begin{array}{l}\text { Crohn's dis- } \\
\text { ease }(n=52)\end{array}$ \\
\hline Age, median (range), years & $47(20-62)$ & $42(19-72)$ \\
\hline Male, n (\%) & $17(56.7)$ & $23(44.2)$ \\
\hline $\begin{array}{l}\text { Disease duration, mean } \pm \text { SD } \\
\text { (range), years }\end{array}$ & $10.1 \pm 7.4$ & $12.4 \pm 11.6$ \\
\hline \multicolumn{3}{|l|}{ Disease extension, n (\%) } \\
\hline Proctitis & $1(3.3)$ & \\
\hline Pancolitis & $17(56.7)$ & \\
\hline Left sided & $12(40)$ & \\
\hline Terminal ileum & & $14(26.9)$ \\
\hline Colonic & & $10(19.2)$ \\
\hline Ileocolitis & & $28(53.9)$ \\
\hline \multicolumn{3}{|l|}{ Current medication, $\mathrm{n}(\%)$} \\
\hline None & $0(0)$ & $8(15.4)$ \\
\hline Mesalazine & $10(33.3)$ & $2(3.8)$ \\
\hline Azathioprine/methotrexate & $1(3.3)$ & $5(9.6)$ \\
\hline Anti-TNF & $12(40)$ & $24(46.2)$ \\
\hline Vedolizumab & $6(20)$ & $2(3.8)$ \\
\hline Ustekinumab & & $4(7.7)$ \\
\hline Combination therapy & $1(3.3)$ & $3(5.8)$ \\
\hline Steroids & $0(0)$ & $4(7.7)$ \\
\hline \multicolumn{3}{|l|}{ Clinical activity scores, n (\%) } \\
\hline \multicolumn{3}{|l|}{ Mayo score } \\
\hline$\leq 5$ & 21 & \\
\hline$\geq 5$ & 9 & \\
\hline \multicolumn{3}{|l|}{ CDAI } \\
\hline$\leq 150$ & & 32 \\
\hline$\geq 150$ & & 20 \\
\hline \multicolumn{3}{|l|}{ Endoscopic scores, mean \pm SD } \\
\hline Mayo score & $1.8 \pm 1.3$ & \\
\hline SES-CD & & $8.4 \pm 10.9$ \\
\hline \multicolumn{3}{|l|}{ Laboratory markers, mean \pm SD } \\
\hline C-reactive protein (mg/L) & $7 \pm 7.8$ & $9.4 \pm 13.5$ \\
\hline
\end{tabular}

gates, granulomas, and number of eosinophils, thereby assessing a total of nine histologic features [27]. With this, the score exhibits a total range from 0 to 27 with 0 to 4 corresponding with histologic remission, a score $\geq 5$ with histological active disease, 5 to 9 low activity, 10 to 18 moderate activity, and $\geq 19$ high activity [27].
Patients screened $(n=108)$

Patients excluded $(n=26)$

- poor bowel preparation $(n=5)$

- anastomotic stenosis $(n=4)$

- only sigmoidoscopies $(n=17)$

Patients included $(n=82)$

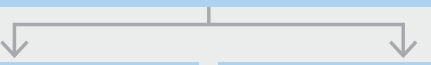

Crohn's disease $(n=52)$

- clinically healed $(n=28)$

- clinically not healed ( $n=24)$

Ulcerative colitis ( $n=30$ )

- clinically healed $(n=10)$

- clinically not healed $(n=20)$

- Fig. 1 Flowchart of the included patients [28].

\section{Statistical analysis}

Parametric data are expressed in this report as the mean and SD, and categorical data are presented as percentages. The primary aim of this study was to assess diagnostic accuracy, sensitivity, and specificity of magnification endoscopy in conjunction with i-scan OE for assessment of histologic inflammation in patients with IBD. Therefore, histopathologic scoring was used as a reference for validation of endoscopic assessment. Correlation between histology scores and endoscopy scores used in the study was calculated using Spearman's correlation approach. Results are presented as correlation coefficient $r$, and $P<0.05$ was considered to be statistically significant. In the post hoc phase, the four endoscopists analysed at least one high-quality image/video of the ROI, represented by the most affected segment. Fleiss' kappa statistics (к) were calculated to measure interobserver agreement, with $>0.75$ indicating very good agreement, 0.4 to 0.75 indicating good agreement, and $<0.4$ indicating poor agreement. As a study of diagnostic accuracy, all statistics are reported according to the STARD guidelines [28].

\section{Results}

\section{Patient characteristics}

In total, 82 consecutively enrolled patients with IBD with a mean disease duration of 11.6 years (UC: 10.1 years, CD: 12.4 years) were included in this study. The study cohort consisted of 40 males (49\%) and 42 females (51\%) with a median age of 43 years (range 19 to 72 years). Further demographic details, clinical patient characteristics and current medication are summarized in $>$ Table 1. According to the STARD guidelines [28], a flowchart of the included patients is represented in $>$ Fig. 1. 


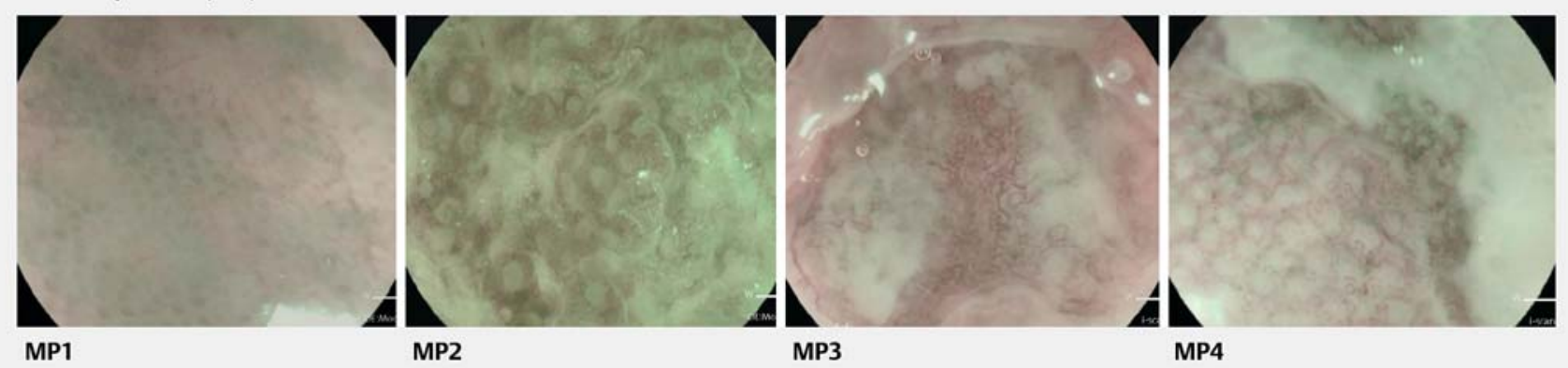

\section{Vascular pattern (VP)}

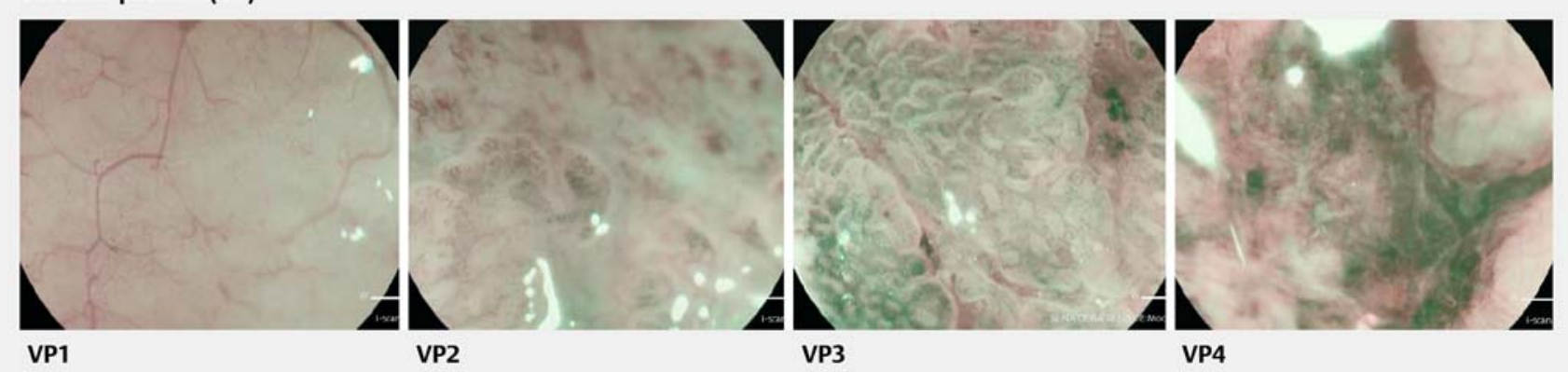

- Fig. 2 Mucosal and vascular pattern grading in patients with UC and CD in the colon under magnification endoscopy with i-scan OE. Upper row: Grading of the mucosal pattern was based on a scoring from 1 to 4 , as outlined in $>$ Table 2. Lower row: Grading of the vascular pattern was based on a scoring from 1 to 4 , as outlined in $>$ Table 2 .

- Table 2 i-scan OE magnification endoscopy score for UC and CD in the colon [19].

\begin{tabular}{|l|l|}
\hline Score & Characteristics \\
\hline Vascular pattern (VP) score \\
\hline VP Grade 1 & $\begin{array}{l}\text { Round vessels surrounding crypts, isolated spiral } \\
\text { vessels }\end{array}$ \\
\hline VP Grade 2 & Crowded/tortuous/serrated vessels \\
\hline VP Grade 3 & Dilated vessels with intramucosal bleeding \\
\hline VP Grade 4 & Destroyed vessels with luminal bleeding \\
\hline Mucosal pattern (MP) score \\
\hline MP Grade 1 & Round/dilated/absent crypts \\
\hline MP Grade 2 & Round crypts with microerosion \\
\hline MP Grade 3 & Dilated/elongated crypts with erosion \\
\hline MP Grade 4 & Hyperplastic crypts with necrosis and ulcers \\
\hline Overall score & Mucosal + vascular pattern score \\
\hline
\end{tabular}

\section{Magnification scores under i-scan OE}

In vivo assessment of inflammatory activity in patients with UC was done according to a previously described score assessing the vascular and mucosal pattern under magnification endoscopy in combination with i-scan OE [19]. Endoscopic scoring of inflammatory activity in the colonic segments of patients with
CD was likewise performed according to this score $(\triangleright$ Table 2,

- Fig. 2) For assessment of disease activity in the terminal ileum of patients with $C D$, a new magnification score under i-scan OE was developed incorporating mucosal and vascular alterations. For assessment of mucosal pattern (MP) in the terminal ileum, deformation of the villi as well as presence of erosions, ulcers, and/or necrosis were evaluated, resulting in an MP score ranging from 1 to 4 , where a score of 1 was represented by normal, leaf-shaped villi, a score of 2 by deformed club-shaped villi, a score of 3 by club-shaped villi with erosions and a score of 4 by deformed villi with ulceration and necrosis. Similarly, vascular pattern (VP) was graded from 1 to 4 based on absence of vessels or a vascular arcade around the villous' membrane (VP= $1)$, spiral/serrated vessels inside the villous $(V P=2)$ and presence of either intravillous ( $V P=3$ ) or intervillous haemorrhages $(\mathrm{VP}=4)$. Hence, assessing MP and VP in the terminal ileum resulted in a combined overall score (OS) from 2 to 8 ( $\downarrow$ Table 3 , - Fig.3). Examples of active disease under magnification endoscopy in UC and CD can be found as video files ( $\downarrow$ Video 1, - Video 2).

During endoscopic scoring, 24 patients (29.3\%) were graded with complete mucosal and vascular healing, defined as a cumulative score (MP + VP) of 2 under magnification endoscopy with i-scan OE, 18 patients (21.9\%) with light inflammation, 21 patients $(25.6 \%)$ with moderate inflammation and 19 patients (23.2\%) with severe inflammation. A summary of the assigned vascular and mucosal i-scan OE magnification scores in the total cohort is shown in $>$ Table 4 . 


\section{Mucosal pattern (MP)}
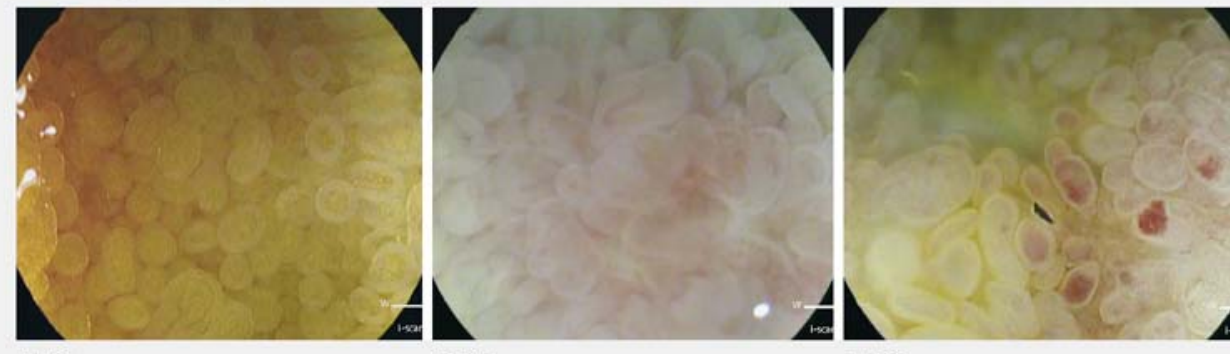

MP3

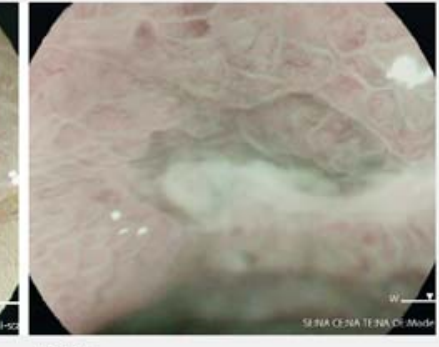

MP1

MP2

MP4

\section{Vascular pattern (VP)}
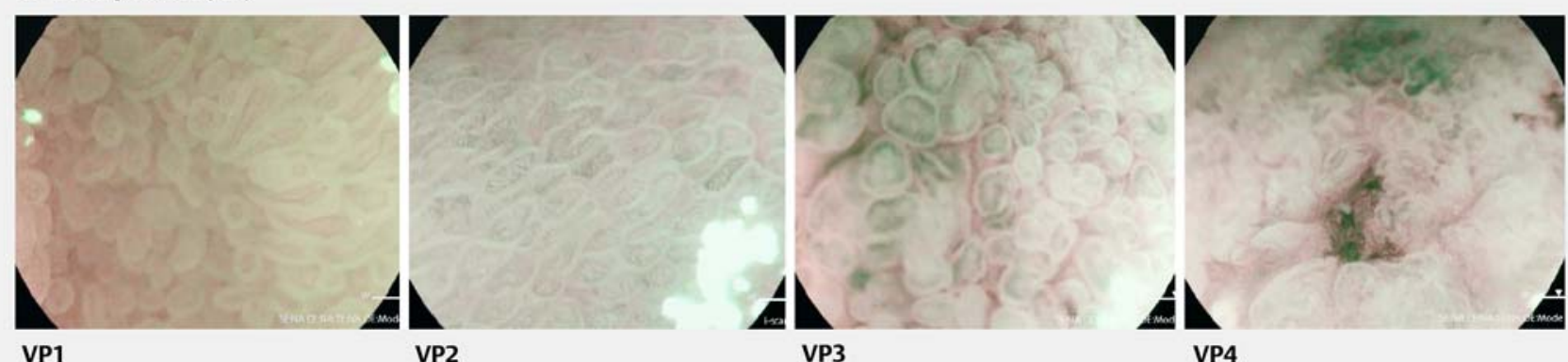

- Fig. 3 Mucosal and vascular pattern grading in patients with CD in the terminal ileum under magnification endoscopy with i-scan OE. Upper row: Grading of the mucosal pattern was based on a scoring from 1 to 4 , as outlined in $\triangleright$ Table 3. Lower row: Grading of the vascular pattern was based on a scoring from 1 to 4 , as outlined in $>$ Table 3 .

- Table 3 i-scan OE magnification endoscopy score for CD in the terminal ileum.

\begin{tabular}{|c|c|}
\hline Score & Characteristics \\
\hline \multicolumn{2}{|c|}{ Vascular pattern (VP) score } \\
\hline VP Grade 1 & No vessels visible or vascular arcade within the villi \\
\hline VP Grade 2 & Spiral/serrated vessels inside the villi \\
\hline VP Grade 3 & Intravillous haemorrhage \\
\hline VP Grade 4 & $\begin{array}{l}\text { Intervillous haemorrhage and/or vascular distortion } \\
\text { with areal bleeding }\end{array}$ \\
\hline \multicolumn{2}{|c|}{ Mucosal pattern (MP) score } \\
\hline MP Grade 1 & Normal villi \\
\hline MP Grade 2 & $\begin{array}{l}\text { Deformed, hypertrophied villi with irregular size, } \\
\text { shape and distance }\end{array}$ \\
\hline MP Grade 3 & Deformed, hypertrophied villi with erosions \\
\hline MP Grade 4 & $\begin{array}{l}\text { Deformed, hypertrophied villi with ulcerations and } \\
\text { necrosis }\end{array}$ \\
\hline Overall score & Mucosal + vascular pattern score \\
\hline
\end{tabular}

\section{Correlation with histopathology}

Overall, magnification i-scan OE scoring showed significant correlation with the Mayo ES and the SES-C with an $r=0.9(95 \% \mathrm{Cl}$ : $0.8-0.95, P<0.05)$ and an $r=0.92(95 \% \mathrm{Cl}: 0-86-0-95, P<$
0.05), respectively. However, of 32 patients as classified as having achieved mucosal healing under standard WLE (UC: Mayo $E S \leq 1, C D$ : absence of erosions or ulcerations), 8 patients (25\%) exhibited persistent abnormal VP and MP scoring as assessed by magnification endoscopy with i-scan OE. Respective vascular and mucosal magnification scores under i-scan OE of the patients with mucosal healing under WLE are shown in - Table 5 .

In contrast, of 24 patients who had complete mucosal and vascular healing under magnification endoscopy in combination with i-scan $\mathrm{OE}(\mathrm{MP}+\mathrm{VP}=2)$, none showed signs of inflammation in histopathological assessment.

When compared with RHI, scoring under magnification endoscopy with i-scan OE showed very strong correlation with an $r=0.83$ (95\% Cl: 0.66-0-92, $P<0.05)$. Correspondingly, histologic scoring with $\mathrm{NHI}$ also showed strong and significant correlation with scoring under magnification endoscopy with an $r$ $=0.78$ (95\% Cl: 0.57-0-89, P<0.05). In CD, assessing MP and VP under magnification endoscopy with i-scan OE showed a strong correlation with histopathological scoring according to the modified RI ( $r=0.74,95 \% \mathrm{Cl}: 0.58-0-85, P<0.05)$.

Diagnostic sensitivity, specificity, and accuracy of assessing inflammation with magnification endoscopy together with iscan OE for the different histologic scores as well as correlation with histopathology in comparison to WLE are summarized in $\checkmark$ Table 6 .

Of note, 2 of 30 UC patients exhibited inflammation under WLE of the terminal ileum, i.e. backwash ileitis. In these pa- 


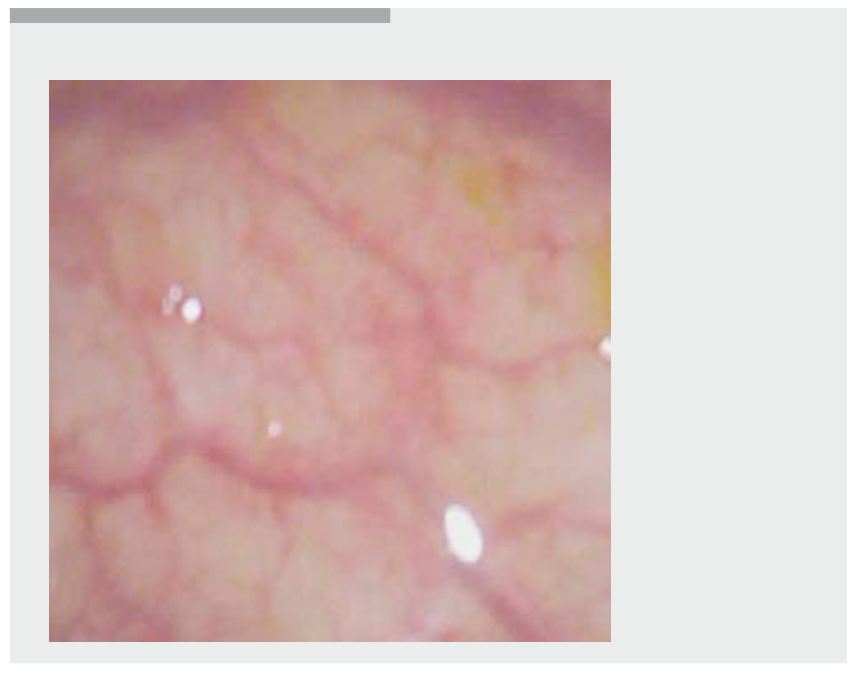

$\checkmark$ Video 1 Active UC during magnification endoscopy. Note the presence of spiral/serrated vessels around the colonic crypts.

- Table 4 Magnification endoscopy scoring in the whole IBD cohort.

\begin{tabular}{|l|l|l|}
\hline Score & Mucosal pattern & Vascular pattern \\
\hline 1 & 36 & 31 \\
\hline 2 & 12 & 15 \\
\hline 3 & 12 & 25 \\
\hline 4 & 22 & 11 \\
\hline
\end{tabular}

- Table 5 Patients with mucosal healing under WLE $(n=32)$ and their corresponding mucosal and vascular pattern scoring with magnification endoscopy and i-scan OE.

\begin{tabular}{|l|l|l|}
\hline Score & Mucosal pattern & Vascular pattern \\
\hline 1 & 25 & 24 \\
\hline 2 & 5 & 5 \\
\hline 3 & 2 & 3 \\
\hline 4 & 0 & 0 \\
\hline
\end{tabular}

tients, inflammatory assessment under magnification endoscopy with i-scan OE correlated perfectly with histopathology.

\section{Interobserver agreement}

Interobserver agreement between the three endoscopists who scored the collected images/videos was calculated, culminating in a $\mathrm{K}$ statistic of $0.76(95 \% \mathrm{Cl} 65-84)$. According to the previously described interpretation of kappa values, this indicates "very good" agreement between the endoscopists for assessing inflammatory activity under magnification endoscopy in combination with i-scan OE.

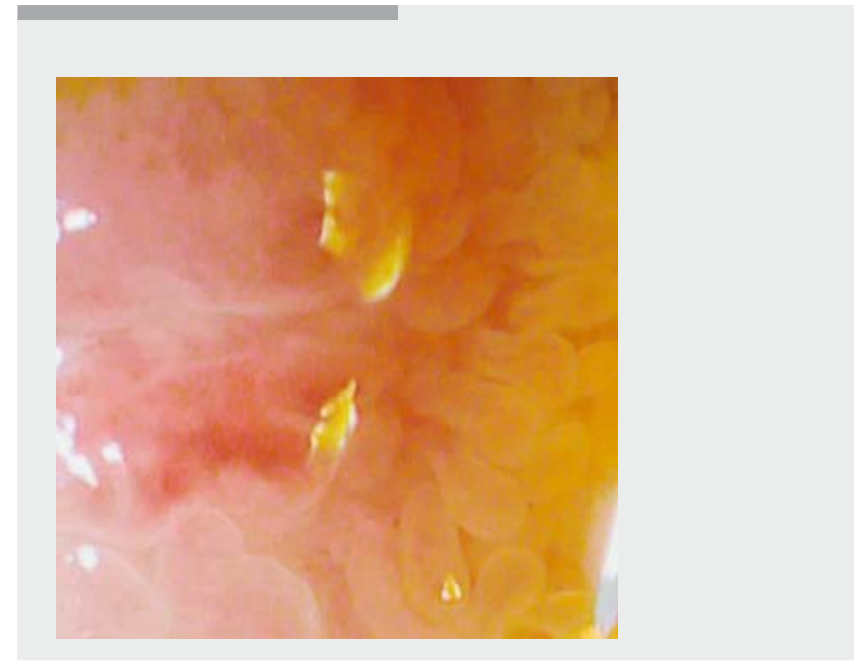

Video 2 Active CD during magnification endoscopy. Note the presence of intervillous hemorrhages.

\section{Discussion}

In this study, we showed that magnification endoscopy in conjunction with i-scan OE as a novel virtual chromoendoscopy (VC) technique shows strong correlation with histologic inflammation in patients with IBD. Of note, of 32 patients who were considered to have achieved mucosal healing on conventional WLE, $25 \%$ had persistent microscopic inflammation on histopathology, and even more importantly, this residual microscopic inflammation correlated to an abnormal mucosal or vascular pattern under magnification endoscopy in conjunction with i-scan OE. In contrast, of the 24 patients whp had complete mucosal and vascular healing under magnification endoscopy, none exhibited microscopic inflammation on histopathological scoring.

These findings have several important implications: First, although mucosal healing as assessed by conventional WLE is an established treatment goal that is associated with improved long-term outcome in patients with IBD [2], accumulating evidence suggests histological remission might serve as an even better predictor of patient outcomes, especially with regard to corticosteroid use and hospitalization rates than mucosal healing $[13,14]$. Therefore, the international organization for the study of inflammatory bowel diseases (IOIBD) has already proposed histologic healing as another key treatment goal in IBD patients [15]. Given these considerations, it seems clear that endoscopic techniques that can precisely assess microscopic inflammation can facilitate and improve patient care and decision-making about medical therapy. Second, the most commonly used current scoring systems, such as Mayo ES in UC or SES-CD, mainly implement mucosal abnormalities such as presence of erosions or ulcerations, mucosal friability, presence of stenosis or surface affected by any lesions while vascular abnormalities are typically underrepresented within these scores. Altered vasculature, however, is a frequently observed finding in patients with IBD with certain clinical relevance. A variety of studies have shown that mucosal angiogenesis not only plays 


\begin{tabular}{|c|c|c|c|c|}
\hline & \multicolumn{4}{|l|}{ Ulcerative colitis } \\
\hline & ME/i-scan OE and RHI & ME/i-scan OE and NHI & WLE and RHI & WLE and NHI \\
\hline Accuracy, \% & 82 & 71 & 57 & 54 \\
\hline Sensitivity, \% (95\% CI) & $88.2(62-98)$ & $76.5(50-92)$ & $69.2(39-90)$ & $68(41-88)$ \\
\hline Specificity, \% (95\% CI) & $63.6(32-88)$ & $63.6(32-88)$ & $33(13-61)$ & $33(11-65)$ \\
\hline \multirow[t]{3}{*}{ Correlation r (95\% Cl) } & $0.83(0.66-0.92)$ & $0.78(0.57-0.89)$ & $0.67(0.39-0.83)$ & $0.62(0.32-0.82)$ \\
\hline & \multicolumn{4}{|l|}{ Crohn's disease } \\
\hline & \multicolumn{2}{|l|}{$M E / i-s c a n ~ O E$ and $m R I$} & \multicolumn{2}{|l|}{ WLE and $\mathrm{mRI}$} \\
\hline Accuracy, \% & \multicolumn{2}{|l|}{67} & \multicolumn{2}{|l|}{55} \\
\hline Sensitivity, \% (95\% Cl) & \multicolumn{2}{|l|}{$76.2(53-91)$} & \multicolumn{2}{|l|}{$52.2(31-73)$} \\
\hline Specificity, \% (95\% CI) & \multicolumn{2}{|l|}{$60.7(41-78)$} & \multicolumn{2}{|l|}{$57.6(37-76)$} \\
\hline Correlation r $(95 \% \mathrm{Cl})$ & \multicolumn{2}{|l|}{$0.74(0.58-0.85)$} & \multicolumn{2}{|l|}{$0.68(0.49-0.81)$} \\
\hline
\end{tabular}

an important role in the initiation and perpetuation of chronic intestinal inflammation commonly associated with IBD [2931] but also that increased vascular density in IBD directly correlates with disease severity [32]. More recent endoscopic scores, such as The Ulcerative Colitis Endoscopic Index of Severity (UCEIS) [33], already address this and incorporated alterations of the vascular pattern with a distinction between normal, patchy, and complete obliteration for grading disease activity. However, this is an evaluation that is based on conventional assessment with WLE while at the same time, a recent study provides evidence that only magnification endoscopy in conjunction with NBI unmasked a certain vascular pattern that served as an important endoscopic predictive feature of clinical recurrence in patients with UC [18]. Third, consistent with previous reports $[18,19]$, our results corroborate that magnification endoscopy in conjunction with optical chromoendoscopy can render endoscopic diagnosis of inflammatory activity more precisely and closer to histology in patients with UC and now illustrate at the same time that the same approach can be readily applied in patients with CD likewise to successfully assess microscopic inflammation.

Interestingly, in our study 2 of 30 patients with UC exhibited inflammation under WLE of the terminal ileum, i. e. backwash ileitis. In these patients, inflammatory assessment under magnification endoscopy with i-scan OE was made according to the introduced score ( $>$ Fig. 3, $>$ Table 3 ) and correlated perfectly with histopathology. In the future, magnification endoscopy in combination with i-scan OE might be useful to detect backwash ileitis otherwise not visible under WLE in patients with UC.

Advanced endoscopic imaging techniques for inflammatory assessment in IBD can be roughly divided into dye-based and dye-less chromoendoscopy [34,35] and results for assessing IBD-associated intestinal inflammation have been summarized recently in a systematic review [6]. One of the earliest prospective studies in 30 patients with UC identified specific vascular patterns under NBI which were associated with acute inflam- matory cell infiltrates, goblet cell depletion, and basal plasmacytosis on histopathology, thereby providing evidence that NBI during colonoscopy may allow a more precise grading of mucosal alterations [31]. The role of NBI combined with magnification endoscopy for assessment of histologic inflammation has also been assessed. In this regard, it has been shown that magnification endoscopy together with NBI can identify branch-like structures and the vascular architecture of the domes in Peyer's patches in IBD patients [36] and that presence of a certain vascular pattern indicates histopathological activity and is associated with disease relapse [18]. Apart from studies on NBI, another recent study also demonstrated that magnification endoscopy together with optical enhancement can accurately assess histologic inflammation in 41 patients with UC [19]. In this report, we not only confirmed these results but also extend this to patients with $C D$. In their totality, our results together with the studies discussed above indicate that optical assessment of the mucosa in patients with IBD is feasible at almost histological level using magnification endoscopy and optical chromoendoscopy. Therefore, these techniques represent a powerful addition to the diagnostic armamentarium of the colonoscopist screening patients with IBD for their disease activity that hold the potential to reduce the number of physical biopsies required during screening colonoscopies for monitoring disease activity in patients with IBD.

Although this prospective study included a large number of patients with IBD, potential limitations also need to be addressed, one of which is the fact that all colonoscopies were performed by a single experienced endoscopist at a single center. However, this is partly due to the fact that magnification endoscopy is currently not widely available in Europe and clearly, multicenter and multi-operator studies will be needed to corroborate these findings. Further potential limitations should also be mentioned. This is a pilot study with only a restricted amount of patients and larger cohorts are needed to further reaffirm the ability of magnification endoscopy in combination with i-scan 
OE for detection of microinflammation in IBD patients. Initial studies on new technologies in academic hospitals are known to demonstrate better outcomes as compared to studies in routine clinical practice outside academic centers [37], therefore, performance of this technique might deteriorate when used by community based endoscopists. Correlation for a scoring system rated by the developers might be prone to a certain bias, and correlation might be decreased when used by endoscopists outside our expert group. An additional limitation of this study is that histologic inflammation might not be fully represented by and correlated with endoscopically observed inflammation. In patients with $C D$, inflammation is known to vary even in the same colonic segment, making a direct comparison between histologic and endoscopic inflammation challenging. More specifically, transmural inflammation might not be represented in our magnification score for CD. In our study, the most severe enteric segment was evaluated as representative of underlying inflammation.

\section{Conclusion}

In summary, our results show that i-scan OE together with magnification endoscopy shows strong correlation with histologic inflammation in both patients with UC and with CD, therefore rendering the endoscopic diagnosis more precise, especially in patients who exhibit mucosal healing under conventional WLE.

\section{Acknowledgements}

The authors acknowledge the help of the endoscopy staff of the Ludwig Demling Endoscopy Center at the University Hospital Erlangen.

\section{Competing interests}

None

\section{References}

[1] Peyrin-Biroulet L, Sandborn W, Sands BE et al. Selecting Therapeutic Targets in Inflammatory Bowel Disease (STRIDE): Determining Therapeutic goals for treat-to-target. Am J Gastroenterol 2015; 110 : $1324-1338$

[2] Neurath MF, Travis SP. Mucosal healing in inflammatory bowel diseases: a systematic review. Gut 2012; 61: 1619-1635

[3] Rutgeerts P, Vermeire S, Van Assche G. Mucosal healing in inflammatory bowel disease: impossible ideal or therapeutic target? Gut 2007; 56: $453-455$

[4] Sandborn W], Hanauer S, Van Assche G et al. Treating beyond symptoms with a view to improving patient outcomes in inflammatory bowel diseases. J Crohns Colitis 2014; 8: 927 - 935

[5] Colombel JF, Rutgeerts P, Reinisch W et al. Early mucosal healing with infliximab is associated with improved long-term clinical outcomes in ulcerative colitis. Gastroenterology 2011; 141: 1194 - 1201

[6] Kiesslich R, Neurath MF. Advanced endoscopy imaging in inflammatory bowel diseases. Gastrointest Endosc 2017; 85: 496 - 508
[7] Travis SP, Higgins PD, Orchard T et al. Review article: defining remission in ulcerative colitis. Aliment Pharmacol Ther 2011; 34: 113-124

[8] Peyrin-Biroulet L, Ferrante M, Magro F et al. Results from the 2nd Scientific Workshop of the ECCO. I: Impact of mucosal healing on the course of inflammatory bowel disease. J Crohns Colitis 2011; 5: 477 483

[9] Froslie KF, Jahnsen J, Moum BA et al. Mucosal healing in inflammatory bowel disease: results from a Norwegian population-based cohort. Gastroenterology 2007; 133: 412-422

[10] lacucci M, Ghosh S. Mucosal healing - how deep is enough? Dig Dis 2016; 34: $160-164$

[11] Danese S, Fiorino G, Angelucci E et al. Narrow-band imaging endoscopy to assess mucosal angiogenesis in inflammatory bowel disease: a pilot study. World J Gastroenterol 2010; 16: 2396-2400

[12] lacucci M, Fort Gasia M, Hassan C et al. Complete mucosal healing defined by endoscopic Mayo subscore still demonstrates abnormalities by novel high definition colonoscopy and refined histological gradings. Endoscopy 2015; 47: 726-734

[13] Bryant RV, Burger DC, Delo J et al. Beyond endoscopic mucosal healing in UC: histological remission better predicts corticosteroid use and hospitalisation over 6 years of follow-up. Gut 2016; 65: 408-414

[14] Frieri G, Galletti B, Di Ruscio M et al. The prognostic value of histology in ulcerative colitis in clinical remission with mesalazine. Therap Adv Gastroenterol 2017; 10: 749-759

[15] Bryant RV, Winer S, Travis SP et al. Systematic review: histological remission in inflammatory bowel disease. Is 'complete' remission the new treatment paradigm? An IOIBD initiative J Crohns Colitis 2014; 8: $1582-1597$

[16] Mosli MH, Feagan BG, Zou G et al. Reproducibility of histological assessments of disease activity in UC. Gut 2015; 64: 1765-1773

[17] Walsh AJ, Bryant RV, Travis SP. Current best practice for disease activity assessment in IBD. Nat Rev Gastroenterol Hepatol 2016; 13: 567 579

[18] Sasanuma S, Ohtsuka K, Kudo SE et al. Narrow band imaging efficiency in evaluation of mucosal healing/relapse of ulcerative colitis. Endosc Int Open 2018; 6: E518-E523

[19] lacucci M, Kiesslich R, Gui X et al. Beyond white light: optical enhancement in conjunction with magnification colonoscopy for the assessment of mucosal healing in ulcerative colitis. Endoscopy 2017; 49: $553-559$

[20] Satsangi ], Silverberg MS, Vermeire S et al. The Montreal classification of inflammatory bowel disease: controversies, consensus, and implications. Gut 2006; 55: $749-753$

[21] Peyrin-Biroulet L, Panes J, Sandborn WJ et al. Defining disease severity in inflammatory bowel diseases: current and future directions. Clin Gastroenterol Hepatol 2016; 14: 348 - 354 e317

[22] Schroeder KW, Tremaine W], Ilstrup DM. Coated oral 5-aminosalicylic acid therapy for mildly to moderately active ulcerative colitis. A randomized study. N Engl J Med 1987; 317: 1625-1629

[23] Daperno M, D'Haens G, Van Assche G et al. Development and validation of a new, simplified endoscopic activity score for Crohn's disease: the SES-CD. Gastrointest Endosc 2004; 60: 505-512

[24] Mosli MH, Feagan BG, Zou G et al. Development and validation of a histological index for UC. Gut 2017; 66: 50 - 58

[25] Marchal-Bressenot A, Salleron J, Boulagnon-Rombi C et al. Development and validation of the Nancy histological index for UC. Gut 2017; 66: $43-49$

[26] Riley SA, Mani V, Goodman MJ et al. Microscopic activity in ulcerative colitis: what does it mean? Gut 1991; 32: 174-178

[27] Knieling F, Neufert C, Hartmann A et al. Multispectral optoacoustic tomography for assessment of Crohn's disease activity. N Engl J Med 2017; 376: $1292-1294$ 
[28] Cohen JF, Korevaar DA, Altman DG et al. STARD 2015 guidelines for reporting diagnostic accuracy studies: explanation and elaboration. BMJ Open 2016; 6: e012799

[29] Danese S, Sans M, de la Motte $C$ et al. Angiogenesis as a novel component of inflammatory bowel disease pathogenesis. Gastroenterology 2006; 130: $2060-2073$

[30] Deban L, Correale C, Vetrano S et al. Multiple pathogenic roles of microvasculature in inflammatory bowel disease: a Jack of all trades. Am J Pathol 2008; 172: $1457-1466$

[31] Kudo T, Matsumoto T, Esaki M et al. Mucosal vascular pattern in ulcerative colitis: observations using narrow band imaging colonoscopy with special reference to histologic inflammation. Int ] Colorectal Dis 2009; 24: 495-501

[32] Cromer WE, Mathis JM, Granger DN et al. Role of the endothelium in inflammatory bowel diseases. World J Gastroenterol 2011; 17: 578 593
[33] Travis SP, Schnell D, Krzeski P et al. Developing an instrument to assess the endoscopic severity of ulcerative colitis: the Ulcerative Colitis Endoscopic Index of Severity (UCEIS). Gut 2012; 61: 535-542

[34] Rath T, Tontini GE, Neurath MF et al. From the surface to the single cell: Novel endoscopic approaches in inflammatory bowel disease. World J Gastroenterol 2015; 21: 11260 - 11272

[35] Waldner M], Rath T, Schurmann S et al. Imaging of mucosal inflammation: current technological developments, clinical implications, and future perspectives. Front Immunol 2017; 8: 1256

[36] Hiyama S, lijima H, Shinzaki S et al. Narrow band imaging with magnifying endoscopy for Peyer's patches in patients with inflammatory bowel disease. Digestion 2013; 87: 269-280

[37] Rees C], Rajasekhar PT, Wilson A et al. Narrow band imaging optical diagnosis of small colorectal polyps in routine clinical practice: the Detect Inspect Characterise Resect and Discard 2 (DISCARD 2) study. Gut 2017; 66: 887-895 